\title{
ORIGINAL ARTICLE \\ Preoperative renal scar as a risk factor of postoperative metabolic acidosis following ileocystoplasty in patients with neurogenic bladder
}

\author{
T Mitsui, K Moriya, T Kitta, M Kon and K Nonomura
}

Objectives: We investigated relation of preoperative renal scar to incidence of postoperative metabolic acidosis following ileocystoplasty in patients with neurogenic bladder.

Patients: Thirty patients with neurogenic bladder, who underwent ileocystoplasty, were enrolled in the present study. Median age at ileocystoplasty was 13.9 years and median follow-up period after ileocystoplasty was 8.2 years. Metabolic acidosis was defined based on the outlined criteria: base excess $(B E)$ is less than $0 \mathrm{mmoll}^{-1}$. Preoperative examination revealed that no apparent renal insufficiency was identified in blood analysis, although preoperative ${ }^{99 m}$ Tc-DMSA scintigraphy indicated abnormalities such as renal scar in 14 patients (47\%). Incidence of postoperative metabolic acidosis was compared between patients with and without preoperative renal scar, which may reflect some extent of renal tubular damage.

Results: Postoperative metabolic acidosis was identified in 13 patients (43\%). Incidence of postoperative metabolic acidosis was significantly higher in patients with renal scar $(11 / 14,79 \%)$ compared with patients without renal scar $(2 / 16,13 \%$; $P<0.01)$. Particularly, all eight patients who had bilateral renal scars showed metabolic acidosis postoperatively. Compared with patients without preoperative renal scar, $\mathrm{pH}(P<0.05)$ and $\mathrm{BE}(P<0.01)$ were significantly lower postoperatively in patients with preoperative renal scar. However, there was no significant difference in $\mathrm{PCO}_{2}$. Hyperchloremia was observed in each patient with or without preoperative renal scar.

Conclusion: Incidence of postoperative metabolic acidosis was significantly implicated in preoperative renal scar. If renal abnormalities are preoperatively identified in imaging tests, we need to care patients carefully regarding metabolic acidosis and subsequent comorbidities following ileocystoplasty.

Spinal Cord (2014) 52, 292-294; doi:10.1038/sc.2013.175; published online 28 January 2014

Keywords: metabolic acidosis; base excess; renal scar; augmentation; bowel; neurogenic bladder

\section{INTRODUCTION}

In severe lower urinary tract dysfunction such as neurogenic bladder, clean intermittent catheterization and anticholinergic medication have been the initial treatment to protect the upper urinary tract and kidneys and to achieve socially acceptable urinary continence. Currently, in addition to anticholinergic mediation, botulinum toxin $^{1,2}$ and beta-3 agonists $^{3}$ are available as a second-line pharmacotherapy to treat lower urinary tract dysfunction such as neurogenic bladder in some cases. However, surgical interventions are also necessary in some cases as options to store an appropriate volume of urine with a low filling pressure, when these medical treatments fail. Surgical interventions include bladder augmentation using bowel tissue. Bladder augmentation has been a useful intervention to achieve these goals.

However, complications following the use of bowel tissue for bladder augmentation are well documented. These complications include metabolic acidosis, mucous production, bladder stone, bladder tumor, rupture of tissue for augmentation and so on. ${ }^{4}$ Of these complications, postoperatively major concerns following the use of intestinal tissue for bladder augmentation is possible metabolic acidosis, because it can cause subsequent comorbidities such as growth retardation in children and osteoporosis. ${ }^{5}$
In the present study, we investigated the relation of preoperative renal scar to incidence of postoperative metabolic acidosis following ileocystoplasty in patients with neurogenic bladder.

\section{PATIENTS AND METHODS}

We retrospectively reviewed medical charts in patients with neurogenic bladder, who underwent prior ileocystoplasty for bladder augmentation, and total 30 patients, consisting of 15 males and 15 females, were enrolled in the present study. None of the patients had any history of other operations on the renal tract that can affect renal function. Urinary tract infections occurred in all patients at least once and the lower urinary tract was managed by intermittent catheterization preoperatively.

Patient's characteristics were shown in Table 1. Median age at ileocystoplasty was 13.9 years (3.1-47.2) and follow-up period after ileocystoplasty was 2.7-23.4 years (median: 8.2 years). Original diseases for lower urinary tract dysfunction were meningomyelocele in 24 patients (80\%), anorectal malformations in 2 patients (7\%), transverse myelitis in 1 patient and unknown in 3 patients (10\%). Preoperative examination revealed that no apparent renal insufficiency was identified in blood analysis. Ureteral reflux was preoperatively identified in 26 ureters of 17 patients (57\%), and it persisted in 4 ureters of 3 patients (10\%) postoperatively. Preoperative ${ }^{99 \mathrm{~m} T c-D M S A}$ scintigraphy indicated that abnormalities such as renal scar, which reflect some extent of renal tubular damage and lead to renal impairment, ${ }^{6}$ were identified in 14 


\section{Table 1 Patient characteristics}

\begin{tabular}{lc}
\hline Gender (males/females) & $15 / 15$ \\
Age at surgery & Median 13.9 years $(3.1-47.2)$ \\
Follow-up period after surgery & Median 8.2 years $(2.7-23.4)$ \\
Original diseases & \\
$\quad$ Meningomyelocele & 24 pts $(80 \%)$ \\
Anorectal malformation & 2 pts $(7 \%)$ \\
Transverse myelitis & 1 pt $(3 \%)$ \\
Unknown & 3 pts $(10 \%)$ \\
Preoperative reflux & 17 pts $(57 \%) 26$ ureters \\
Postoperative reflux & 3 pts $(10 \%) 4$ ureters \\
Preoperative renal scars & 14 pts $(47 \%)$ \\
\hline
\end{tabular}

Abbreviation: Pts, patients.

patients (47\%), including 8 patients who had bilateral renal scars. All renal scars were considered to be because of urinary tract infection. Postoperatively, all patients underwent routine laboratory evaluation of complete blood count, blood urea nitrogen, creatinine, electrolytes and venous blood gases including $\mathrm{pH}$, base excess (BE), bicarbonate $\left(\mathrm{HCO}_{3}{ }^{-}\right)$and $\mathrm{PCO}_{2}$.

Metabolic acidosis was defined based on the outlined criteria: less than $0 \mathrm{mmoll}^{-1}$ of BE. Incidence of postoperative metabolic acidosis was compared between patients with and without preoperative renal scar, which may reflect some extent of renal tubular damage. Also, incidence of postoperative metabolic acidosis was investigated regarding other backgrounds, such as gender, age at surgery, follow-up period and length of bowel segment (Table 2).

Statistical analysis was performed using $\chi^{2}$ test and unpaired Student's $t$-test. $P$ value less than 0.05 was considered significant.

\section{RESULTS}

Postoperative metabolic acidosis was identified in 13 patients (43\%), including 2 patients who had renal insufficiency postoperatively. Postoperative metabolic acidosis was independent on gender, age at surgery, follow-up period or length of bowel segment (Table 2). Incidence of postoperative metabolic acidosis was significantly higher in patients with renal scar: $11(79 \%)$ of 14 patients, whereas only 2 patients (13\%) did not have renal scar, had metabolic acidosis postoperatively (Figure $1, P<0.01$ ). Particularly, all eight patients who had bilateral renal scars showed postoperative metabolic acidosis. Compared with patients without preoperative renal scar, postoperative $\mathrm{pH}$ (Figure 2a, $P<0.05$ ) and $\mathrm{BE}$ (Figure 2b, $P<0.01$ ) were significantly lower in patients with preoperative renal scar. Postoperative $\mathrm{HCO}_{3}{ }^{-}$was also significantly lower in patients with preoperative renal scar $\left(24.9 \pm 3.3 \mathrm{mmoll}^{-1}\right)$ compared with patients without preoperative renal scar $\left(26.4 \pm 1.6 \mathrm{mmoll}^{-1} ; P<0.05\right)$. However, there was no significant difference in $\mathrm{PCO}_{2}$. Hyperchloremia was observed in each patient with or without preoperative renal scar.

\section{DISCUSSION}

Medical charts of patients with neurogenic bladder, who underwent prior ileocystoplasty for bladder augmentation, were retrospectively reviewed regarding postoperative metabolic acidosis, and it was identified in $43 \%$. Incidence of postoperative metabolic acidosis was significantly higher in patients with renal scar, particularly in patients with renal scars in the bilateral kidneys, which may reflect some extent of renal tubular damage. However, postoperative metabolic acidosis was not implicated in gender, age at surgery, follow-up period or length of bowel segment.

In ileocystoplasty on bladder augmentation, a hyperchloremic metabolic acidosis may occur, because ammonium $\left(\mathrm{NH}_{4}{ }^{+}\right)$and chloride $\left(\mathrm{Cl}^{-}\right)$are reabsorbed in segment of the ileum exposed to urine. ${ }^{4,7}$ The net absorption of $\mathrm{NH}_{4}{ }^{+}$for a proton $\left(\mathrm{H}^{+}\right)$is the major
Table 2 Incidence of metabolic acidosis and other factors

\begin{tabular}{|c|c|c|}
\hline Metabolic acidosis (+) (13 pts) & Metabolic acidosis (-) (17 pts) & P-value \\
\hline \multicolumn{3}{|l|}{ Gender (male/female) } \\
\hline $4 \mathrm{pts} / 9 \mathrm{pts}$ & $11 \mathrm{pts} / 6 \mathrm{pts}$ & 0.139 \\
\hline \multicolumn{3}{|l|}{ Age at surgery (years) } \\
\hline $13.4 \pm 6.9$ & $15.9 \pm 10.3$ & 0.385 \\
\hline \multicolumn{3}{|l|}{ Follow-up period (years) } \\
\hline $9.5 \pm 5.3$ & $10.8 \pm 7.4$ & 0.402 \\
\hline \multicolumn{3}{|l|}{ Length of bowel segment (cm) } \\
\hline $37.8 \pm 7.1$ & $37.1 \pm 7.0$ & 0.527 \\
\hline
\end{tabular}

Abbreviation: Pts, patients.

Mean \pm s.d.

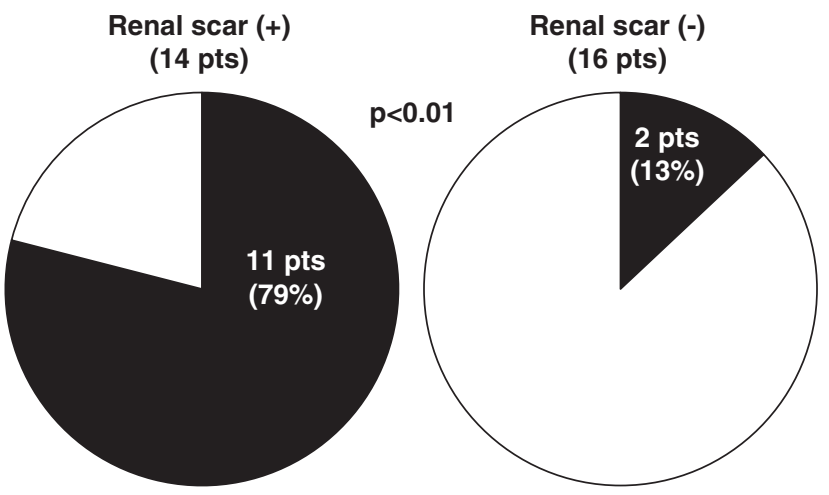

Figure 1 Incidence of metabolic acidosis and renal scar. Incidence of postoperative metabolic acidosis was significantly higher in patient with renal scar.

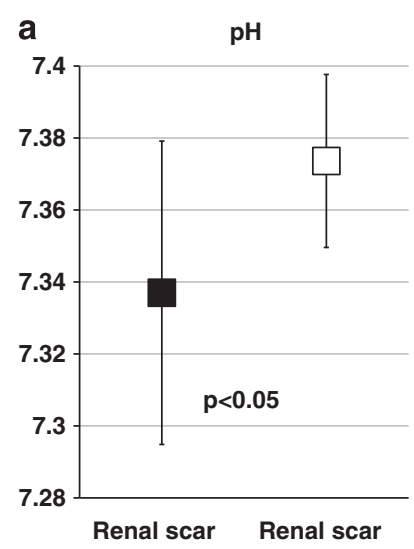

$(+)$

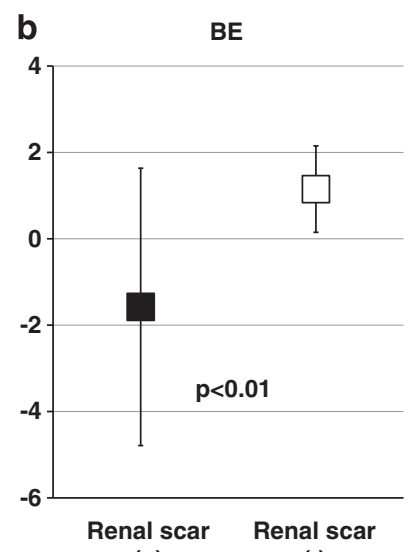

$(+)$
$(-)$

Figure 2 (a) $\mathrm{pH}$ associated with renal scar. Compared with patients without preoperative renal scar, $\mathrm{pH}$ was significantly lower in patients with renal scar. Mean \pm s.d. (b) BE-associated renal scar. BE was significantly lower in patients who had preoperative renal scar. Mean \pm s.d.

contributor to increased acid load and metabolic acidosis, and $\mathrm{HCO}_{3}{ }^{-}$is secreted in exchange of $\mathrm{Cl}^{-}$. As a consequence, the presence of ileal segment for bladder augmentation implies a chronic acid load. Metabolic acidosis after bladder augmentation may induce 
subsequent comorbidities such as alternations in patterns of growth and bone density changes. ${ }^{8,9}$ Alkalinizing therapy is often performed as an effective treatment in restoring acid-base balance, if metabolic acidosis is identified after bladder augmentation.

In patients with adequate renal function, the kidneys are able to process the increased acid load and seldom do clinical consequences arise. In fact, longitudinal data from preoperative and postoperative assessments in patients with normal serum creatinine did not show metabolic acidosis, and other clinical factors beyond the surgery could be implicated in incidence of postoperative metabolic acidosis. ${ }^{10}$ Regarding renal function after enterocystoplasty, urinary diversion could not deteriorate renal function, although chronic retention or infection caused by inadequate catheterization and poor compliance in urodynamics may deteriorate renal function. ${ }^{11,12}$ Actually, two patients, who showed renal insufficiency and metabolic acidosis postoperatively in the present study, already had multiple renal scars and a border value of preoperative serum creatinine in renal function preoperatively, which could be implicated in deterioration of renal function after bladder augmentation.

Even patients without apparent renal insufficiency also showed metabolic acidosis postoperatively, and most of these patients had renal scar preoperatively, particularly in the bilateral kidneys, in the present study. Renal scar reflects some extent of renal tubular damage, and it is well known that the proximal and distal tubules in the kidneys actually serve maintaining acid-base homeostasis. Even though metabolic acidosis is not identified preoperatively in patients who have normal renal function with renal scar, a chronic acid load after ileocystoplasty may induce to appear metabolic acidosis without deterioration of renal function. Thus, preoperative renal scar, particularly in the bilateral kidneys, is one of the risk factors of postoperative metabolic acidosis after ileocystoplasty.

Considering the complications of urine contact with the bowels, length of segment, retained time length of urine and concentration of solutes in urine are also important. In the previous studies, metabolic hyperchloremic acidosis predominates when longer segment is used for reservoir construction. ${ }^{13}$ Then, it is also reported that incidence of metabolic complications is greater in patients with continent reservoirs of the bowels compared with conduit, ${ }^{13,14}$ which could be associated with length of segment and retained time length of urine. Further, the absorptive properties of the intestinal segment of bladder augmentation possibly change with time when it contacts with urine. Previous studies revealed that histology of intestinal segment in contact with urine showed mucosal atrophy and decreased villose height, ${ }^{15}$ which indicated reduction of absorptive capacity. ${ }^{16}$ In the present study, there was no significant correlation between postoperative metabolic acidosis and follow-up period or length of bowel segment (Table 2). Actually, we could not find cutoff values of follow-up period or length of bowel segment as a risk factor of metabolic acidosis. This is one of the limitations of the present study as it just includes a small number of patients. Then, we could not evaluate the frequency and interval of catheterization for retained time length of urine, frequency of urinary tract infections or concentration of solutes in urine in the present study. Further studies are necessary to investigate the mechanism of postoperative metabolic acidosis.

\section{CONCLUSIONS}

Incidence of postoperative metabolic acidosis was significantly implicated in preoperative renal scar. Particularly, preoperative renal scars in the bilateral kidneys are one of risk factors of postoperative metabolic acidosis after ileocystoplasty. If renal abnormalities are preoperatively identified in imaging tests, we need to care patients carefully regarding metabolic acidosis and subsequent comorbidities following ileocystoplasty.

\section{DATA ARCHIVING}

There were no data to deposit.

\section{CONFLICT OF INTEREST}

The authors declare no conflict of interest.

1 Ginsberg D, Gousse A, Keppenne V, Sievert KD, Thompson C, Lam W et al. Phase 3 efficacy and tolerability study of onabotulinumtoxinA for urinary incontinence from neurogenic detrusor overactivity. J Urol 2012; 187: 2131-2139.

2 Cruz F, Herschorn S, Aliotta P, Brin M, Thompson C, Lam W et al. Efficacy and safety of onabotulinumtoxinA in patients with urinary incontinence due to neurogenic detrusor overactivity: a randomised, double-blind, placebo-controlled trial. Eur Urol 2011; 60: 742-750.

3 Yamaguchi 0 . Latest treatment for lower urinary tract dysfunction: therapeutic agents and mechanism of action. Int J Urol 2013; 20: 28-39.

4 Stein R, Schroder A, Thuroff JW. Bladder augmentation and urinary diversion in patients with neurogenic bladder: non-surgical considerations. J Pediatr Urol 2012: 8: 145-152.

5 Kraut JA, Madias NE. Consequences and therapy of the metabolic acidosis of chronic kidney disease. Pediatr Nephrol 2011; 26: 19-28.

6 Chen MJ, Cheng HL, Chiou YY. Risk factors for renal scarring and deterioration of renal function in primary vesico-ureteral reflux children: a long-term follow-up retrospective cohort study. PLoS One 2013; 8: e57954.

7 Van der Aa F, Joniau S, Van Den Branden M, Van Poppel H. Metabolic changes after urinary diversion. Adv Urol 2011; 2011: 764325.

8 Mingin GC, Nguyen HT, Mathias RS, Shepherd JA, Glidden D, Baskin LS. Growth and metabolic consequences of bladder augmentation in children with myelomeningocele and bladder exstrophy. Pediatrics 2002; 110: 1193-1198.

9 Feng $\mathrm{AH}$, Kaar S, Elder JS. Influence of enterocystoplasty on linear growth in children with exstrophy. J Urol 2002; 167: 2552-2555.

10 Adams RC, Vachha B, Samuelson ML, Keefover-Hicks A, Snodgrass WT. Incidence of new onset metabolic acidosis following enteroplasty for myelomeningocele. J Urol 2010; 183: 302-305

11 Fontaine $E$, Leaver $R$, Woodhouse $C R$. The effect of intestinal urinary reservoirs on renal function: a 10-year follow-up. BJU Int 2000; 86: 195-198.

12 Jonsson O, Olofsson G, Lindholm E, Törnqvist H. Long-time experience with the Kock ileal reservoir for continent urinary diversion. Eur Urol 2001; 40: 632-640.

13 Lockhart JL, Davies R, Persky L, Figueroa TE, Ramirez G. Acid-base changes following urinary tract reconstruction for continent diversion and orthotopic bladder replacement. J Urol 1994; 152: 338-342.

14 Poulsen AL, Steven K. Acid-base metabolism after bladder substitution with the ilea urethral Kock reservoir. Br J Urol 1996; 78: 47-53.

15 Deane AM, Woodhouse CR, Parkinson MC. Histological changes in ileal conduits. J Urol 1984; 132: 1108-1111.

16 Akerlund S, Forssell-Aronsson E, Jonsson O, Kock NG. Decreased absorption of $22 \mathrm{Na}$ and $36 \mathrm{Cl}$ in ileal reservoirs after exposure to urine. An experimental study in patients with continent ileal reservoirs for urinary or fecal diversion. Urol Res 1991; 19: 249-252. 